thorough removal of the glands checks the progress of the disease, although it will certainly recur. Some of the later cases also demonstrate with what safety and facility enormous quantities of glands may be removed from the neck owing to the existence of the mobility which has been insisted upon.

CASE 1.-J. G., aged 4r, was admitted to the London Hospital in April, 1888. Two years ago he noticed a lump in the middle of the posterior triangle of the neck on the right side. After an interval of twelve months the anterior triangle on the left side.

Condition in 1888. - There are five or six considerably enlarged glands in the posterior triangles on both sides of the neck and one in the anterior triangle on the left side. The glands are all firm, distinct from each other, and freely movable beneath the skin and on the deeper structures. The spleen is not enlarged.

Treatment.-No improvement followed the administration of arsenic during a period of two months. At two successive operations all the during a period of two months. At two successive operations all the shlarged glands were removed. With two exceptions all the glands surfaces were smooth and the section pale and homogeneous, without trace of softening or caseation. The patient had all the appearance of trace of softening or caseation. The patic

Condition in June, r894.- He has now a fresh crop of glands on both sides of the neck. They are situated chiefly in the parotid and submaxillary regions and behind the ears. one is observed above the right maxicle and one in the right axilla. The glands were removed at two successive operations, and they all shelled out quite readily.

Condition in 1895.-This patient was again admitted in November, 85, with very many considerably enlarged glands extending down both sides of the neck, chietly along the course of the large vessels. There was one in the right axilla. The glands still continued movable. No appreciable enlargement of the liver or spleen His general health and appearance remained good. general health and appearance remained good. He was treated with injections of Coley's fluid, which had then recently come into use, fifteen injections being given, extending over a period of three
months. At first a slight reaction tcok place, but latterly the reaction
was very violent, the temperature in some instances reaching was very violent, the temperature in some instances reaching ro $^{\circ}$ to ${ }^{106^{\circ}}$. The enlargement certainly subsided somewhat, but the treatment could not be considered in any respect curative. On some occasions injections were made into the enlarged glands, and this resulted in a just before his discharge from the hospital showed per cubic $\mathrm{mm}$.

$$
\begin{array}{lllr}
\text { Red corpuscles } & \ldots & \ldots & 3,860,000 \\
\text { White corpuscles } & \ldots & \ldots & \text { 5,000 } \\
\text { Hæmoglobin } \ldots & \ldots & \ldots & 75 \text { p. c. }
\end{array}
$$

The red corpuscles were therefore nearly normal, and the white slightly increased, a condition by no means surprising considering the constitutional disturbances and pyrexia to which he had been subjected.

CASE II. - H. L., aged 8, was admitted to the London Hospital, December 16 th, 189 r. This patient had a mass of enlarged glands on the right side of the neck, of a precisely similar character to the foregoing. They were removed. On November $\mathrm{r}_{4}$ th, 1892, he was readmitted with a recurrence on the subclavian triangle on the right side; and the glands were all again removed, being still non-adherent. Another group of enlarged glands was removed in January, ${ }^{8} 89.3$ They occupied the posterior and sub-maxillary triangles on the right side. In May, 1894 , he again returned with enlarged glands on the right side, situated behind the ramus of the lower jaw, in front of the sterno-mastoid, and just above the clavicle in the posterior triangle. All the enlarged glands were removed, and shelled out readily. His general health remained good. Since tinat date I have lost sight of him.

CASE III.-G. R., aged 13 , was admitted to the London Hospital in January, 189o, with a large mass of enlarged glands on the right side of the neck, extending from the lower jaw to the clavicle. They were all non-adherent and freely movable. The enlargement had commenced four years previously. A few small glands were felt in the axillæ but none elsewhere were enlarged. He appeared healthy, not anæmic, and his spleen was not enlarged. A great quantity of glands, twenty to thirty in number, were removed at one sitting from the neck, the sternomastoid muscle being divided transversely, and turned upwards and downwards. All the glands shelled out with great facility. They were smooth, firm, homogeneous on section, and without appearance of softening or caseation. To exclude tuberculosis two guinea-pigs were inoculated in the groin with portions of gland, and, when killed two months later, they were found to be quite healthy.

CASE IV. - J. W., aged 7, was admitted in May, 189o. The mother stated that an enlarged gland appeared below the angle of the jaw on the left side two years ago. The enlargement now affects the glands over the 'whole of the left side of the neck, those on the right gide being only shotty. The patient is fairly nourished, and appears in good health. Thirty-two glands were removed from the left side of the neck, the largest attaining the size of a bantam's egg; all were easily shelled out. In this case, again, guinea-pigs were inoculated without result.

REFERENCES.
Acad. des Sciences, June i7th, r895. 2 since writing the above I have succeeded in finding this patient. He applied for admission at another succeeded in finding this patient. He applied for admission at another hospital, where all the enlarged glands were removed except two His natural ; general appearance healthy.

An adventurous lady doctor, Mlle. Zurcher, is, it is stated, about to start for Abyssinia, where she proposes to practise her profession. She has been recommended to the Negus by an engineer who is presumably a persona yrata at the court of that potentate. Mlle. Zurcher is a native of Zürich, and was in practice for some time at Berne, and afterwards at Tresden.

\section{A CASE OF FACIAL HEMIATROPHY.}

By EUGENE S. YONGE, M.B.,

Assistant Medical Officer, Manchester Hospital for Consumption and Diseases of the Throat.

THE patient, a woman aged $4 \mathrm{I}$, first noticed something wrong with her face twenty-one years ago, when she had an attack of intense facial neuralgia on both sides, lasting, with short intermissions, about six months. Her friends remarked at this period that the right half of her face had become slightly different from the left, and the asymmetry steadily grew more noticeable until nine or ten years ago, since when little or no change has been apparent. At the present time the atrophy is found to involve the right malar bone to the greatest extent, the corresponding frontal coming next in order. The skin on the affected side is furrowed, wrinkled, and thinned, and the superficial veins stand out in bold relief. A wellmarked vertical furrow on the forehead separates the healthy

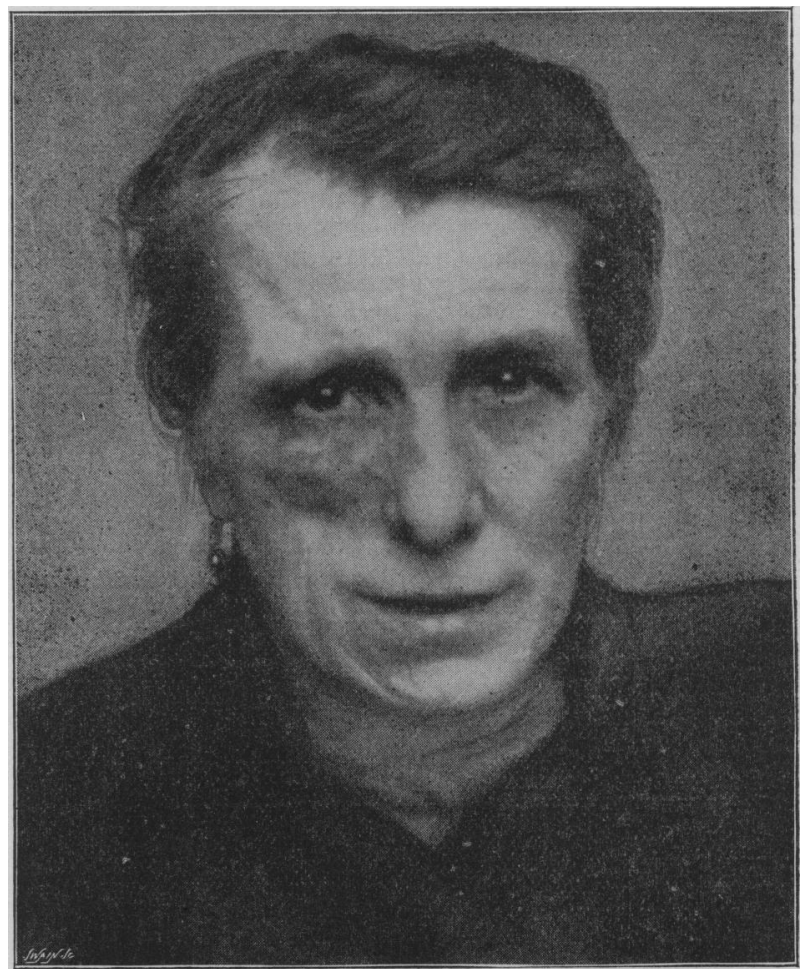

from the diseased portion. The jaw, which is somewhat atrophied, is shorter and shallower on the right side, and that half of the face, in consequence, does not appear as long as the opposite half. The cartilage of the nose is wasted unilaterally. The muscles evidence no change in size, voluntary power, or electrical excitability. The corresponding half of the tongue is slightly atrophied; sensation in the cutaneous area on the right half of the face is unchanged, and neither the temperature nor the secretion of saliva or tears is deranged.

I am much indebted to Dr. Haring, Honorary Assistant Physician to the Manchester Hospital for Consumption, for kind assistance in connection with this case.

The SPanish Wounded.-The wounded soldiers coming from Cuba and the Philippines are carried by the steamship (Spanish) companies as second-class passengers, and at times first-class cabins are offered to them if unoccupied. The railway companies are ready to carry the wounded as second class passengers, although the State allowance is only thirdclass fare. On board the steamers the soldiers are fed and treated, inder the doctors, in the most liberal manner. The Queen of Spain has duly recognised with thanks the kindness shown by the heads of the various companies to the wounded. 